\title{
Pneumatosis intestinalis and spontaneous perforation associated with drug toxicity in oncologic patients: a case series
}

\author{
S. Brocchi ${ }^{1}$, A. Parmeggiani ${ }^{1}$, C. Gaudiano ${ }^{1}$, C. Balacchi ${ }^{1}$, M. Renzulli ${ }^{1}$, N.Brandi $^{1}$, F.G. Dall'Olio ${ }^{2}$, K. Rihawi ${ }^{2}$, A. \\ Ardizzoni $^{2}$, R. Golfieri ${ }^{1}$
}

(1) Department of Radiology, IRCCS Azienda Ospedaliero, Universitaria di Bologna, Bologna, Italia ; (2) Department of Medical Oncology, IRCCS Azienda OspedalieroUniversitaria di Bologna, Bologna, Italia.

\begin{abstract}
Pneumatosis Intestinalis (PI) is a rare radiological finding defined as the presence of extra-luminal gas within the intestinal wall. Several anti-tumor drugs can induce a damage of the gastrointestinal walls as an adverse effect, causing loss of mucosal integrity and endoluminal gas diffusion, responsible for PI development. We retrospectively analyzed 8 cases of PI detected through radiological imaging in oncologic patients undergoing various therapeutic regimens: five patients were receiving chemotherapy, two molecular targeted therapy (MTT) and one immunotherapy.

Three patients were asymptomatic and pneumatosis was incidentally detected at routinary follow-up CT and then treated conservatively. Five patients presented acute abdomen symptoms and in these cases bowel perforation was the cause of death. Our experience confirms PI and perforation as rare complications of drug toxicity, especially in oncologic patients treated with combinations of different anticancer drugs and documented the second reported case of PI associated with atezolizumab and alectinib single administration. (Acta gastroenterol. belg., 2021, 84, 497-499).
\end{abstract}

Keywords: Medical oncology, pneumatosis intestinalis, immunotherapy, drug therapy, molecular targeted therapy, intestinal perforation.

\section{Introduction}

Pneumatosis Intestinalis (PI) is a rare radiological finding defined as the presence of extra-luminal gas within the intestinal wall, in the submucosa or, more likely, subserosa.

Primary PI is generally asymptomatic and resolves spontaneously ; secondary PI is more common and can be associated with trauma, lung diseases (e.g. COPD and emphysema), autoimmune diseases, gastrointestinal conditions such as mesenteric ischemia, bowel obstruction and necrosis, IBD and infection. Moreover, secondary PI may result from iatrogenic causes including diagnostic, surgical and endoscopic procedures and drug administration: in particular, many antitumor drugs can damage the gastrointestinal walls as an adverse effect, thus leading to a loss of mucosal integrity and to a consequential endoluminal gas diffusion, responsible for PI development $(1,2)$. Even if PI is a non-specific finding, it must be always monitored, as it may prelude to more serious complications, such as intestinal perforation, severe peritonitis and even death (3).

In this study, we retrospectively analyzed 8 cases of PI detected through radiological imaging in oncologic patients reporting tumor type, type of treatment and its duration, the presence or absence of clinical symptoms before the scan as well as patients' outcome and whether the drug was interrupted.

\section{Case series}

This retrospective case series entailed a radiology database search from January 2016 through April 2019, which found 8 oncologic patients whose radiology reports mentioned pneumatosis intestinalis, pneumoperitoneum, portal pneumatosis and bowel perforation. Imaging and medical records were reviewed to report clinical features, treatment type and its duration, presence or absence of clinical symptoms before the scan, drug discontinuation, patients' outcome and radiological findings. The cohort included 8 patients, 3 women and 5 men (mean age 65.5 years), without any confounding factors and history for pneumatosis or perforation.

Overall, 6 patients had a lung cancer diagnosis, one patient had renal carcinoma and one patient had oesophageal cancer. Five patients were receiving chemotherapy, two patients molecular targeted therapy and one patient immunotherapy (see Table 1 for details). Three patients were asymptomatic and pneumatosis was incidentally detected at routinary CT and then treated conservatively. Five patients presented acute abdomen symptoms with average survival of 10.4 days after CT and in all these patients bowel perforation was the cause of death.

Imaging findings at CT included pneumatosis $(5 / 8)$ (Figure 1), ascites (1/8), pneumoperitoneum (7/8) (Figure 2), portal pneumatosis (1/8); the site of intestinal perforation was identified in three patients.

\section{Discussion}

PI is usually a benign disease that resolves spontaneously, but occasionally it can lead to surgical complications such as bowel obstruction, intestinal perforation,

Correspondence to: Stefano Brocchi, MD, Department of Radiology, IRCCS Azienda Ospedaliero-Universitaria di Bologna, via Albertoni 15, Bologna, Italia. Phone: +39 0512142307.

E-mail: stefano.brocchi85@gmail.com

Submission date : 19/10/2020

Acceptance date : 02/12/2020 
Table 1.

\begin{tabular}{|c|c|c|c|c|c|c|c|}
\hline Case & Gender & Age & Type of cancer & Therapeutic regimen & $\begin{array}{c}\text { Localization of } \\
\text { pneumatosis }\end{array}$ & $\begin{array}{c}\text { Clinical } \\
\text { presentation }\end{array}$ & Outcome \\
\hline 1 & $\mathrm{M}$ & 62 & Lung adenocarcinoma & carboplatin + paclitaxel & Colon & Asymptomatic & Conservative treatment \\
\hline 2 & $\mathrm{M}$ & 54 & Oesophageal cancer & cisplatin +5 -fluororuracil & Rectum & Acute abdomen & Death \\
\hline 3 & $\mathrm{~F}$ & 72 & Lung adenocarcinoma & paclitaxel & Colon & Acute abdomen & Death \\
\hline 4 & $\mathrm{~F}$ & 64 & Large-cell lung carcinoma & carboplatin + etoposide & Colon & Acute abdomen & Death \\
\hline 5 & $\mathrm{M}$ & 76 & Lung adenocarcinoma & paclitaxel & Colon & Acute abdomen & Death \\
\hline 6 & $\mathrm{M}$ & 65 & Lung adenocarcinoma & atezolizumab & Ileum & Asymptomatic & Conservative treatment \\
\hline 7 & $\mathrm{~F}$ & 57 & Lung adenocarcinoma & alectinib & Ileum & Asymptomatic & Conservative treatment \\
\hline 8 & $\mathrm{M}$ & 74 & Renal cell carcinoma & pazopanib & Colon & Acute abdomen & Death \\
\hline
\end{tabular}

Abbreviations : $\mathrm{M}$ : male, $\mathrm{F}$ : female.

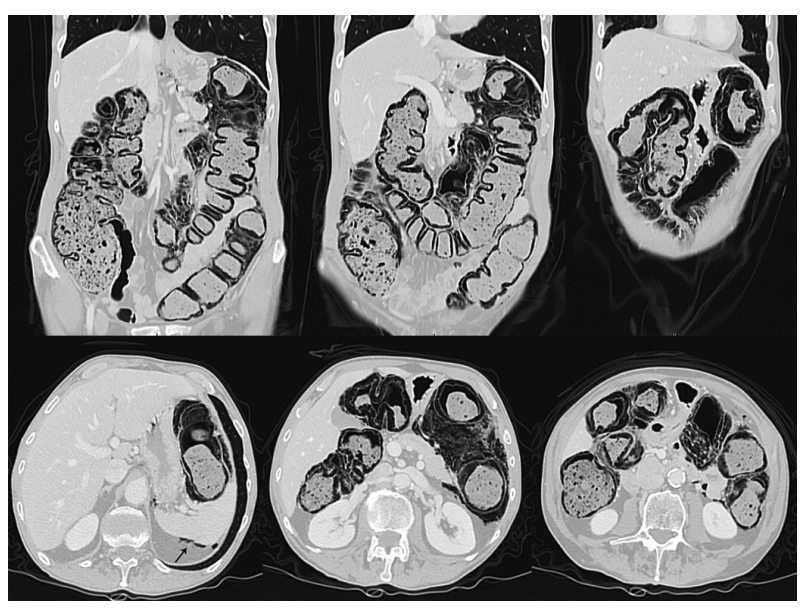

Figure 1. - Post-contrast CT scan in portal venous phase with lung parenchyma window : Multiplanar Reconstruction (MPR) images in coronal and axial plane show the presence of extensive colonic pneumatosis intestinalis and free air in left sub-diaphragmatic space (arrow).

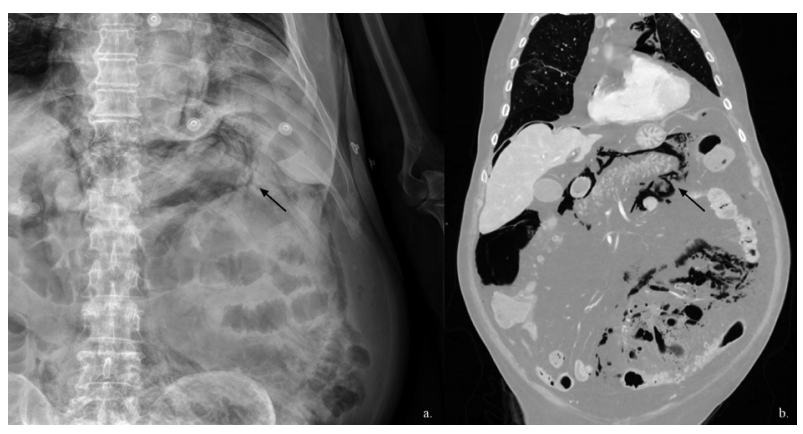

Figure 2. - The image compares diffuse pneumoperitoneum in the abdominal quadrants (black arrows) in the anteroposterior projection of conventional radiography (a) and in CT reconstruction in the coronal plane with lung parenchyma window (b).

volvulus, and gastrointestinal bleeding thus leading to urgent surgery due to their high mortality risk (4).

Three theories of PI's pathogenesis have been identified: according to the mechanical one, PI is due to an increase of intraluminal pressure; the pulmonary theory implies that chronic lung diseases can cause alveolar rupture, with air migration to the bowel, and the bacterial theory, suggests that aerogenic bacteria are responsible for gas development within the gastrointestinal wall from fermentation processes (3).

It has already been observed that chemotherapy can induce gastrointestinal damage, like ileus, enterocolitis, secretory diarrhea and, in particular, PI and gastrointestinal perforation.

In particular, since chemotherapeutic agents interrupt the cell cycle at various levels, inducing apoptosis and blocking cell replication, they can have a great impact on gastrointestinal mucosal cells, which rapidly divide and constantly undergo regeneration processes (5).

Therefore, such drugs can lead to alteration of gastrointestinal mucosa with integrity loss, leading to pneumatosis, intestinal necrosis and perforation ; moreover, their anti-proliferative properties block the repair processes of the damaged mucosa, further favoring PI development (6). Cases of PI and gastrointestinal perforation due to chemotherapeutic drugs have already been reported in literature, both for single and combined regimens (7).

The new molecular target agents act on specific molecular pathways or vital proteins involved in growth regulation of cancer cells, thus protecting the normal body cells and reducing side effects (5).

In particular, alectinib is an anaplastic lymphoma kinase (ALK) inhibitor used in patients with nonsmallcell lung cancer, harbouring ALK rearrangements. Even if ALK inhibitors have been associated with various adverse effects, such as visual disturbances, nausea and vomiting (8), to the best of our knowledge our patient represents the second case of PI associated to alectinib administration described in literature (9).

Pazopanib is a tyrosine kinase inhibitor that specifically targets the vascular endothelial growth factor (VEGF) which promotes angiogenesis and is often overexpressed in cancer cells. As such drugs target kinases which are not always differentially expressed by endothelial cells, they might affect not only the activated tumour endothelium but also normal vasculature, thus leading to specific toxicities (10). Assoun et al. noted that $42.2 \%$ of oncologic patients treated with VEGF inhibitors developed gastrointestinal adverse effects, mainly as digestive perforations requiring an admission to an intensive care unit as life-threatening condition, including a case specifically engaging the administration 
of pazopanib (11). The actual etiopathogenesis is still unclear: angiogenesis inhibitors might damage the structure and function of gastrointestinal vasculature, resulting in ischemic perforation of the normal bowel.

Immunotherapy represents a highly promising cancer therapeutic approach with remarkable antitumor responses and limited side effects. Immune checkpoint inhibitors include atezolizumab, a programmed cell death protein-1 ligand (PD-L1) which blocks the interaction between PD-1 and PD-L1 and PD-L2, thus increasing local tumor-specific immune response (12).

The main adverse event related to PD-L1 inhibitor is an immune-mediated colitis, whose pathogenesis is still not entirely understood and that can quickly progress to lifethreatening conditions like toxic megacolon, peritonitis, bowel perforation, and death (13). At present, only one case of intestinal perforation due to atezolizumab administration has been reported in literature (14), while in our case the patient had developed an asymptomatic PI incidentally detected during routinary follow-up CT.

In conclusion, radiologists should be aware that PI and perforation are rare complications of cancer treatments. This is particularly important with immunotherapy, since it can have side effects that could not always be easy to detect and manage, as evidenced by our experience. At the same time, clinicians should not underestimate an abdominal symptomatology in patients undergoing oncological treatments and should not be afraid to promote targeted radiological investigations for early diagnosis and management. Due to the possible evolution in bowel perforation and death, PI should be immediately reported and strictly monitored, especially in oncologic patients.

\section{Conflict of interest and financial support}

None.

\section{References}

1. TORRES US, FORTES CDFM, SALVADORI PS, TIFERES DA, D IPPOLITO G. Pneumatosis From Esophagus to Rectum: A Comprehensive Review Focusing on Clinico-Radiological Differentiation Between Benign and Life-Threatening Causes. Semin Ultrasound CT MR. 2018 Apr, 39(2) : $167-182$.

2. DE RUYCK E, BAERT F, GHILLEBERT G. An uncommon cause of coffee ground emesis : necrotizing enteritis with pneumatosis intestinalis. Acta Gastroenterol. Belg. 2018 Jan-Mar, 81(1) :111-112.

3. KHALIL PN, HUBER-WAGNER S, LADURNER R, KLEESPIES A, SIEBECK M, MUTSCHLER et al. Natural history, clinical pattern, and surgical considerations of pneumatosis intestinalis. Eur. J. Med. Res. 2009 Jun $18, \mathbf{1 4}(6): 231-9$.

4. BRIGHI M, VACCARI S, LAURO A, D'ANDREA V, PAGANO N, MARINO IR, et al. "Cystamatic" Review: Is Surgery Mandatory for Pneumatosis Cystoides Intestinalis? Dig. Dis. Sci. 2019 Oct, 64(10) : 27692775 .

5. GRAY EJ, DARVISHZADEH A, SHARMA A, GANESHAN D, FARIA SC, LALL C. Cancer therapy-related complications in the bowel and mesentery: an imaging perspective. Abdom. Radiol. (NY). 2016 Oct, 41(10) : 2031-47.

6. CHEN E, ABU-SBEIH H, THIRUMURTHI S, MALLEPALLY N, KHURANA S, WEI D et al. Clinical characteristics of colitis induced by taxane-based chemotherapy. Ann. Gastroenterol. 2020 Jan-Feb, 33(1) : 59-67. doi: 10.20524/aog.2019.0431. Epub 2019 Nov 21.

7. JAYAKODY S, WRIGHT DB, CHIONG C, LIU M, BOUFFLER C, ELKHOURY T. Rectal perforation following paclitaxel and carboplatin chemotherapy for advanced ovarian cancer: a case report and review of the literature. J. Med. Case Rep. 2018 Aug 16, 12(1) : 221.

8. LI G, DAI WR, SHAO FC. Effect of ALK-inhibitors in the treatment of nonsmall cell lung cancer: a systematic review and meta-analysis. Eur. Rev. Med. Pharmacol. Sci. 2017 Aug, 21(15) : 3496-3503.

9. YANAGISAWAA, HAYAMA N, AMANO H, NAKAMURA M, HIRANO S, NAKAMURA S, et al. Crizotinib-induced Rectal Perforation with Abscess. Intern. Med. 2017 Dec 1, 56(23) : 3211-3213.

10. GOTINK KJ, VERHEUL HM. Anti-angiogenic tyrosine kinase inhibitors: what is their mechanism of action? Angiogenesis. 2010 Mar, 13(1) : 1-14.

11. ASSOUN S, LEMIALE V, AZOULAY E. Molecular targeted therapy-related life-threatening toxicity in patients with malignancies. A systematic review of published cases. Intensive Care Med. 2019 Jul, 45(7) : 988-997.

12. ALSAAB HO, SAU S, ALZHRANI R, TATIPARTI K, BHISE K, KASHAW SK, et al. PD-1 and PD-L1 Checkpoint Signaling Inhibition for Cancer Immunotherapy: Mechanism, Combinations, and Clinical Outcome. Front Pharmacol. 2017 Aug 23, $8: 561$.

13. BELLAGUARDA E, HANAUER S. Checkpoint Inhibitor-Induced Colitis. Am. J. Gastroenterol. $2020 \mathrm{Feb}, \mathbf{1 1 5}(2):$ 202-210.

14. ZILLIOUX J, DILIZIA M, SCHAHEEN B, RUSTIN R, KRUPSKI TL. Ileal perforation in the setting of atezolizumab immunotherapy for advanced bladder cancer. Can. J. Urol. 2018 Oct, 25(5) : 9525-9526. 